\title{
Editorial: Contact Interactions in Quantum Mechanics-Theory, Mathematical Aspects and Applications
}

\author{
Manuel Gadella ${ }^{1}$, José Tadeu Lunardi ${ }^{2 \star}$ and Luiz A. Manzoni ${ }^{3}$ \\ ${ }^{1}$ Departamento de Física Teórica, Atómica y Óptica and IMUVA, Universidad de Valladolid, Valladolid, Spain, ${ }^{2}$ Departamento de \\ Matemática e Estatística, Universidade Estadual de Ponta Grossa, Ponta Grossa, Brazil, ${ }^{3}$ Department of Physics, Concordia \\ College, Moorhead, MN, United States
}

Keywords: contact interactions, singular potentials, point interactions, self-adjoint extensions of symmetric operators, regularization and renormalization methods, theory of distributions, solvable models in Quantum Mechanics

Editorial on the Research Topic

Contact Interactions in Quantum Mechanics: Theory, Mathematical Aspects and Applications

Contact interactions refer to a broad range of interactions usually described by potentials with support on a set of dimension lower than the dimension of the ambient space. Typical examples are point potentials given by Dirac deltas, finite or infinite combinations of Dirac deltas or its derivatives, and other types of interactions supported on curves, surfaces, and manifolds. In the last thirty years contact interactions in quantum mechanics have attracted a growing interest, since they provide solvable or quasi solvable models which are very useful for the study of a variety of properties of physical systems in a wide range of applications. For instance, they have been used to approximate results for very short-range potentials, to model several kinds of extra thin structures, to mimic point defects in materials, to study heterostructures, to model impurities in quantum field theory models and, more recently, such potentials played an important role in a reinterpretation of the Casimir effect. Contact interactions may also have unexpected relations with other fields, like group theory.

Mathematically, contact interactions are singular, since they are supported on sets of zero Lebesgue measure. Therefore, to unambiguously define a contact interaction it is necessary to establish the mathematical framework used to address the singularities. One method, inherited from quantum field theory, that is commonly used in the physics literature to deal with the singularities, is to define singular potentials by means of a regularization procedure (often followed by renormalization), that is, as the limit of a sequence of regular functions converging in some sense to the singular potential. The regularization method has been particularly useful in investigating the limit of square potentials and the corresponding point interactions, both in non-relativistic and relativistic quantum mechanics. Another method to properly define a singular interaction uses the theory of self-adjoint extensions (SAE) of symmetric operators. In this case, the wave functions in the domain of the self-adjoint Hamiltonian are completely defined by specifying the boundary conditions they must satisfy at the borders of the singularity. The SAE method provides all the possible self-adjoint extensions allowed for a given symmetric operator and it has been used in a wide variety of models and applications. Yet another approach to define singular interactions is by considering the singular potential (and also the wave function, in some approaches) as a distribution and, by using the apparatus of distribution theory, to define consistently the otherwise ill-defined product of the potential and the wave function in the Schrödinger (or Dirac) wave equation. 
The contributed papers to this Research Topic are original works that use the methods mentioned above (and more) to address not only the theoretical and mathematical aspects of contact interactions, but also their applications to a broad range of fields. Below we present a brief description of these contributions.

Glasser considers a quantum system with known green function and obtains the exact green function for the situation in which two $\delta$-impurities are added to the system. After analyzing various particular cases of the parameters, the author generalizes the result for $N$ such impurities. Kulinskii and Panchenko use the conservation of the current density to obtain the boundary conditions defining a self-adjoint Hamiltonian for the one dimensional Schrödinger operator with spin. These self-adjoint extensions produce point potentials such that the spin is not a constant of motion. The authors also add a physical interpretation to this effect. Dell'Antonio defines contact interactions by boundary conditions at the contact manifold and, by using tools of functional analysis and the gamma convergence, obtains self-adjoint extensions of the free Hamiltonian that correspond to weak and strong contact interactions. The author also illustrates these results in some applications in one, two and three spatial dimensions, which include low and high densities Bose-Einstein condensates and the unitary gas.

Bordag et al. consider a one-dimensional scalar field in the background of a Kronig-Penney potential with a $\delta-\delta^{\prime}$ interaction. They obtain the band structure of the model and compute the nonperturbative vacuum energy, using the spectral Zeta-function method. Rabinovich et al. study band spectra of one dimensional systems containing infinite point singularities, each one consisting of a comb of the Dirac delta and its derivative. The self-adjointness of the Hamiltonians is guaranteed by matching the boundary conditions at each singular point. The authors also provide several numerical examples to illustrate the approach. Erman and Turgut consider Hamiltonians perturbed by singular potentials, with support on points and curves, and address the splitting of the bound state energy levels due to quantum tunneling. They show, using Krein's resolvent approach, that the splitting can be obtained perturbatively under certain conditions.

One dimensional Hamiltonians, with electro-magnetic field potentials localized around a point and depending on coupling constants, are studied by Golovaty. By using a limiting procedure that shrinks the supports of these potentials to a single point, the author obtains a potential of the Dirac delta type plus its derivative, if zero energy resonances for one component of the potential exist. The author also shows that such zero energy resonances are solutions of the Schrödinger equation with non-trivial bounded solutions on the whole real line. By using a similar approach, Zolotaryuk et al. start with multilayer regular potentials in one dimension and, after squeezing them by using suitable limiting procedures, obtain point interactions. The authors use a "point" transistor to illustrate the interpretation of the squeezing process as being due to the application of a controllable bias potential. Calçada et al. revisit the onedimensional Hydrogen atom, which is described by a Coulomb-type long-range potential having a singularity at the origin. The authors use an approach based on the Schwartz theory of distributions and present a systematic study regarding the multiplicity and parity of the bound states and the boundedness of the ground state energy for all the possibilities of self-adjoint parity invariant interactions.

Albeverio et al. consider a two-dimensional system, where the free particle Hamiltonian is perturbed with a harmonic oscillator potential in the $x$ direction, plus a Gaussian potential isotropic in the two dimensions. The resulting Hamiltonian is self-adjoint and bounded from below. By replacing the Gaussian potential in the $x$ direction by a Dirac delta potential the authors obtain a new Hamiltonian, with similar properties to the original one, and also estimate a lower bound for the spectrum. The authors also show that this new Hamiltonian is a limit, in the strong resolvent sense, of a sequence of Hamiltonians of the type studied in the first place. Guilarte et al. present an in-depth investigation of the spectral problem for the Dirac Hamiltonian of one-dimensional electrons and positrons with either electrostatic or position-dependent mass impurities given by a $\delta$-function potential, a necessary analysis for their goal of building a fermionic quantum field theory and computing the Casimir effect for this system. In the framework of a point particle effective field theory, Hayman and Burgess describe a process of particle-conversion mediated by flavor-changing interactions, in which two light scalar particles interact by contact with a heavy point particle. The singularities of the interactions at the worldline of the heavy particle are handled by using regularization and renormalization techniques. The authors also discuss the connection of their model to a model of a single particle dynamics described by a non self-adjoint Hamiltonian.

Salem et al. review two methods based on the theory of selfadjoint extensions and apply them to approach Hamiltonians with a point singularity in $(2+1)$ dimensions. They address the problem of a spin-1/2 charged particle with an anomalous magnetic moment in an Aharonov-Bohm potential in a conic space and apply both the methods to obtain a relationship between the parameter of the self-adjoint extension and the physical parameters of the problem. Erman et al. consider one-dimensional systems decorated with either a Dirac delta or its derivative, in both cases multiplied by a time dependent coefficient, and show that this problem may be solved by using the Laplace transform as an intermediate tool so as to obtain the Green function in either case. Some particular cases are analyzed. Finally, Suchanecki investigates the extensions of time operators acting on spaces of generalized functions associated with $K$-systems as well as the problem of the decomposability of cylindrical elements.

This Research Topic is an up-to-date sample of the main ideas, concepts and methods of the research on contact interactions in quantum mechanics, and received works of some of the most active researchers presently working in this subject. We thank all of them for their valuable contributions.

\section{AUTHOR CONTRIBUTIONS}

All authors listed have made a substantial, direct, and intellectual contribution to the work and approved it for publication. 
Conflict of Interest: The authors declare that the research was conducted in the absence of any commercial or financial relationships that could be construed as a potential conflict of interest.

Copyright (c) 2021 Gadella, Lunardi and Manzoni. This is an open-access article distributed under the terms of the Creative Commons Attribution
License (CC BY). The use, distribution or reproduction in other forums is permitted, provided the original author(s) and the copyright owner(s) are credited and that the original publication in this journal is cited, in accordance with accepted academic practice. No use, distribution or reproduction is permitted which does not comply with these terms. 